DOI: $10.12957 /$ demetra.2015.15317

\title{
Avaliação das temperaturas dos alimentos durante o preparo e distribuição em restaurantes comerciais de Belo Horizonte-MG
}

\section{Evaluation of food temperatures during preparation and distribution in commercial restaurants in Belo Horizonte-MG, Brazil}

\author{
Aline Oliveira Penedo' \\ Renata Barreto de Jesus' \\ Simone das Chagas Ferreira Silva' \\ Marlene Azevedo Magalhães Monteiro' \\ Rita de Cássia Ribeiro! \\ 1 Universidade Federal de Minas Gerais, Curso de \\ Nutrição, Departamento de Nutrição, Escola de \\ Enfermagem. Belo Horizonte-MG, Brasil. \\ Correspondência / Correspondence \\ Marlene Azevedo Magalhães Monteiro \\ Universidade Federal de Minas Gerais \\ Escola de Enfermagem - Departamento de \\ Nutrição \\ Av. Prof ${ }^{0}$ Alfredo Balena, 190 - $3^{0}$ andar - Sala \\ 318 - Bairro Santa Efigênia \\ 30130-100 - Belo Horizonte-MG, Brasil \\ E-mail:marleneaz@enf.ufmg.br; \\ marleneammonteiro@gmail.com
}

\section{Resumo}

Introdução: Devido às mudanças nos hábitos de vida da população, as refeições são cada vez mais realizadas fora do ambiente doméstico. Os estabelecimentos comerciais são locais de grande importância no cotidiano das pessoas; assim, é imprescindível que cuidados para o fornecimento de um alimento seguro sejam adotados. O binômio tempo e temperatura é um dos parâmetros que precisam ser muito bem controlados, a fim de evitar o crescimento microbiano. $\mathrm{O}$ objetivo deste trabalho foi avaliar as temperaturas pós-preparo e durante a distribuição dos alimentos em restaurantes comerciais. Metodologia: Trata-se de estudo observacional descritivo realizado em dez restaurantes comerciais self servisse a quilo da região CentroSul do município de Belo Horizonte-MG. Foram realizadas quatro aferições das temperaturas do prato principal (duas opções), da guarnição (uma opção), do acompanhamento (arroz e feijão) e da salada (duas opções), utilizando-se termômetro de espeto digital, em intervalos de uma hora durante três dias alternados. Os dados foram analisados pelo programa SPSS versão 16.0, pela mediana de cada preparação, e comparados ao preconizado pela Resolução RDC 216/2004 e Portaria CVS 6/1999. Resultados: As temperaturas dos pratos quentes variaram de $43^{\circ} \mathrm{C}$ a $91^{\circ} \mathrm{C}$, e dos pratos frios, de $9^{\circ} \mathrm{C}$ a $24^{\circ} \mathrm{C}$. Sessenta por cento dos restaurantes apresentaram inconformidades quanto à temperatura de distribuição dos alimentos, o que sugere risco de contaminação microbiológica. Conclusão: Torna-se indispensável a implantação de técnicas efetivas de controle de temperatura e capacitação adequada dos profissionais envolvidos no processo de distribuição das refeições.

Palavras-chave: Alimentos. Controle de Qualidade. Temperatura. Restaurantes. 


\section{Abstract}

Introduction: Due to changes in the habits of life, meals are increasingly prepared outside the home environment. Commercial establishments are important places in the daily lives of people, thus it is imperative that care for the provision of safe food is adopted. Time and temperature are parameters that need to be thoroughly controlled to avoid microbial growth. This study aimed to evaluate the temperatures after preparation and exposure of food in commercial restaurants. Method: Observational study conducted in ten descriptive commercial self-service restaurants to pound the south-central region of Belo Horizonte-MG. We analyzed the main course (two options), trim (an option), monitoring (rice and beans) and salad (two options), using digital thermometer skewer "EOF" mark with five measurements of one at a time in three alternate days. Statistical analysis was performed using SPSS version 16.0, using the median found in each preparation to evaluate the temperature distribution of food. Data were compared to RDC 216/2004 and Regulation CVS 6/1999. Results: Temperatures of the hot dishes ranged from $43^{\circ} \mathrm{C}$ to $91^{\circ} \mathrm{C}$, and cold dishes, from $9^{\circ} \mathrm{C}$ to $24^{\circ} \mathrm{C}$; $60 \%$ of restaurants showed non for temperature distribution of food, which suggests risk of microbiological contamination. Conclusion: It is essential to implement effective techniques for temperature control and proper training of the professionals involved.

Key words: Food. Quality Control. Temperature. Restaurants.

\section{Introdução}

As transformações da vida moderna provocaram mudanças significativas na alimentação e nos hábitos alimentares das pessoas, que estão a cada dia diminuindo o número de refeições no ambiente doméstico. ${ }^{1}$ Essas mudanças foram ocasionadas por fatores que perpassam a urbanização, a industrialização, a profissionalização das mulheres, a elevação do nível de vida e de educação, o acesso mais amplo da população ao lazer, a redução do tempo para o preparo e/ou consumo do alimento, as viagens, entre outros fatores. ${ }^{1,2}$ Por isso, torna-se indispensável o aprimoramento do serviço de alimentação, sobretudo em restaurantes comerciais, fazendo-se necessário implantar métodos capazes de controlar a qualidade das refeições em suas diversas dimensões, as higiênicosanitárias, nutricionais e sensoriais. ${ }^{1,3}$ 
A segurança dos alimentos está associada à qualidade higiênico-sanitária, uma vez que patógenos veiculados por alimentos são um dos principais fatores que contribuem para os índices de morbidade nos países da América Latina e do Caribe. ${ }^{3}$ Segundo o Comitê da Organização Mundial da Saúde / Organização das Nações Unidas para Alimentação e Agricultura (WHO/FAO), as doenças causadas pelo consumo de alimentos contaminados são, possivelmente, o maior problema de saúde no mundo contemporâneo. Assim, com o aumento no número de refeições fornecidas, cresce a preocupação com a qualidade dos alimentos para garantir a saúde dos usuários. ${ }^{1,4}$

Segundo dados da Associação Brasileira de Refeições Coletivas (ABERC), as empresas nacionais de serviço de alimentação serviram, em 2011, mais de 16,5 milhões de refeições/dia, sendo que para 2012 o crescimento foi em torno de 8,7\%. A despesa média mensal familiar com alimentação fora do domicílio é de aproximadamente $\mathrm{R} \$ 172,78$, na Região Sudeste, o que representa 37,2\% do orçamento gasto com alimentação. ${ }^{4,5}$

Os restaurantes self service, na categoria comercial, são os mais procurados pelos brasileiros. No entanto, esse tipo de serviço ocupa o segundo lugar no ranking de estabelecimentos com maior ocorrência de doenças transmitidas por alimentos (DTAs). ${ }^{6}$

Para que seja garantida a qualidade das refeições, é indispensável ficar atento ao binômio "tempo e temperatura", fator muito importante na distribuição de refeições. A exposição do alimento a temperaturas inadequadas pode promover o desenvolvimento de agentes patógenos que contribuem para o surgimento de surtos de DTAs. Em vários restaurantes, as preparações ficam expostas no balcão térmico por longo período e, na maioria das vezes, sob temperatura inadequada, o que influencia de forma decisiva no crescimento da atividade microbiana. ${ }^{7}$

O objetivo deste estudo foi avaliar as temperaturas durante o preparo e distribuição dos alimentos em restaurantes comerciais do município de Belo Horizonte-MG.

\section{Metodologia}

Trata-se de estudo observacional descritivo envolvendo dez restaurantes comerciais do tipo selfservice e a quilo da região Centro-Sul de Belo Horizonte, Minas Gerais, escolhidos aleatoriamente e com padrão de cardápio semelhante.

As preparações quentes e frias avaliadas foram prato principal (duas opções), guarnição (uma), acompanhamento (arroz e feijão); e salada (dois tipos). As temperaturas foram aferidas com termômetro digital do tipo espeto no centro da cuba, em três dias alternados, entre os meses de janeiro e fevereiro de 2013, e registrados em formulário preestabelecido. 
As temperaturas foram coletadas em quatro momentos: após o preparo do alimento, no início da distribuição, uma hora após o início da distribuição e no final da distribuição. Os dados coletados foram analisados pelo programa SPSS versão 16.0, a partir do cálculo das temperaturas máximas, mínimas e a mediana. Para avaliar as temperaturas de distribuição dos alimentos, utilizou-se a mediana encontrada em cada preparação e considerou-se o tempo médio de exposição do alimento de três horas.

Também se realizou o teste Kruskal-Wallis, com o intuito de analisar o modo de distribuição da variável, determinando-se assim que a mediana seria o melhor parâmetro de análise a ser utilizado.

Os dados obtidos foram comparados com a Resolução RDC 216/20048 e a Portaria CVS 5/2013, ${ }^{9}$ segundo as quais as preparações quentes devem permanecer a temperaturas superiores a $60^{\circ} \mathrm{C}$, por no máximo seis horas de exposição, e os alimentos frios devem ser submetidos a temperaturas inferiores a $10^{\circ} \mathrm{C}$.

\section{Resultados}

A tabela 1 descreve as temperaturas (mínima, mediana e máxima) encontradas para cada preparação analisada. Pode-se afirmar que houve diferença significativa das temperaturas encontradas nas preparações, tanto da pista quente e fria, e entre os restaurantes $(\mathrm{p}<0,0001)$. As temperaturas dos pratos quentes variaram de $43^{\circ} \mathrm{C}$ a $91^{\circ} \mathrm{C}$, e dos pratos frios permaneceram entre $9^{\circ} \mathrm{C}$ e $24^{\circ} \mathrm{C}$. 
Tabela 1. Temperaturas das preparações quentes e frias de restaurantes comerciais da região Centro-Sul de Belo Horizonte-MG, 2013.

\begin{tabular}{|c|c|c|c|c|c|c|c|c|c|c|c|c|}
\hline Alimentos & Temperatura & R1 & $\mathbf{R 2}$ & R3 & R4 & R5 & R6 & R7 & R8 & R9 & R10 & Valor $\mathbf{p}$ \\
\hline \multirow{3}{*}{$\begin{array}{l}\text { Balcão } \\
\text { Quente }\end{array}$} & Mínima & 74 & 67 & 83 & 69 & 65 & 76 & 68 & 48 & 84 & 49 & \multirow{3}{*}{$<0,0001$} \\
\hline & Mediana & 75 & 74 & 86 & 73 & 69,5 & 81,5 & 72,5 & 55,5 & 86 & 54,5 & \\
\hline & Máxima & 79 & 77 & 88 & 75 & 74 & 85 & 75 & 60 & 88 & 60 & \\
\hline \multirow{3}{*}{ Arroz } & Mínima & 60 & 43 & 66 & 58 & 67 & 55 & 52 & 47 & 72 & 50 & \multirow{3}{*}{$<0,0001$} \\
\hline & Mediana & 69 & 46 & 73,5 & 61,5 & 70,5 & 59 & 58,5 & 56 & 75 & 55 & \\
\hline & Máxima & 75 & 48 & 78 & 65 & 76 & 62 & 65 & 62 & 79 & 61 & \\
\hline \multirow{3}{*}{ Feijão } & Mínima & 60 & 48 & 80 & 77 & 65 & 77 & 76 & 65 & 81 & 62 & \multirow{3}{*}{$<0,0001$} \\
\hline & Mediana & 71,5 & 51 & 86,5 & 78 & 71 & 85 & 82,5 & 69,5 & 86 & 68,5 & \\
\hline & Máxima & 75 & 54 & 89 & 80 & 74 & 88 & 86 & 75 & 90 & 72 & \\
\hline \multirow{3}{*}{ Guarnição } & Mínima & 50 & 50 & 55 & 56 & 56 & 49 & 49 & 48 & 67 & 57 & \multirow{3}{*}{$<0,0001$} \\
\hline & Mediana & 60 & 54,5 & 63 & 59,5 & 60 & 55,5 & 61,5 & 55 & 71 & 59,5 & \\
\hline & Máxima & 63 & 60 & 67 & 68 & 66 & 65 & 70 & 62 & 75 & 65 & \\
\hline \multirow{3}{*}{$\begin{array}{c}\text { Prato } \\
\text { Principal } 1\end{array}$} & Mínima & 68 & 68 & 86 & 77 & 75 & 75 & 51 & 68 & 86 & 68 & \multirow{3}{*}{$<0,0001$} \\
\hline & Mediana & 74 & 74 & 87,5 & 79 & 78 & 78 & 67,5 & 74 & 89 & 75,5 & \\
\hline & Máxima & 76 & 76 & 91 & 86 & 81 & 84 & 87 & 79 & 91 & 79 & \\
\hline \multirow{3}{*}{$\begin{array}{c}\text { Prato } \\
\text { Principal } 2\end{array}$} & Mínima & 59 & 59 & 66 & 65 & 55 & 64 & 51 & 57 & 60 & 51 & \multirow{3}{*}{$<0,0001$} \\
\hline & Mediana & 61,5 & 61,5 & 70,5 & 68,5 & 62 & 71,5 & 63 & 62 & 64,5 & 57 & \\
\hline & Máxima & 65 & 63 & 74 & 77 & 64 & 77 & 69 & 68 & 71 & 63 & \\
\hline \multirow{3}{*}{$\begin{array}{c}\text { Balcão } \\
\text { Frio }\end{array}$} & Mínima & 6 & 6 & 6 & 4 & 6 & 7 & 11 & 3 & 2 & 6 & \multirow{3}{*}{$<0,0001$} \\
\hline & Mediana & 7 & 7 & 8,5 & 6,5 & 7,5 & 9 & 12 & 4 & 3,5 & 8 & \\
\hline & Máxima & 10 & 9 & 10 & 10 & 10 & 11 & 14 & 6 & 5 & 10 & \\
\hline \multirow{3}{*}{ Folhosos } & Mínima & 16 & 11 & 13 & 15 & 9 & 14 & 16 & 10 & 12 & 16 & \multirow{3}{*}{$<0,0001$} \\
\hline & Mediana & 17,5 & 13 & 14,5 & 17 & 12,5 & 16 & 18 & 12,5 & 16 & 18 & \\
\hline & Máxima & 19 & 15 & 18 & 18 & 15 & 18 & 19 & 15 & 17 & 20 & \\
\hline \multirow{3}{*}{ Legumes } & Mínima & 19 & 14 & 17 & 15 & 16 & 12 & 17 & 14 & 17 & 17 & \multirow{3}{*}{$<0,0001$} \\
\hline & Mediana & 2 & 17,5 & 20 & 17 & 18,5 & 13 & 20 & 16,5 & 18 & 20 & \\
\hline & Máxima & 22 & 21 & 22 & 20 & 21 & 20 & 23 & 19 & 22 & 24 & \\
\hline
\end{tabular}


As tabelas 2 e 3 demonstram que as temperaturas das preparações da pista quente e fria não variaram significativamente durante os dias e os diferentes horários de coleta $(p>0,05)$.

Tabela 2. Correlação das medianas por dia das preparações quentes e frias de restaurantes comerciais da região Centro-Sul de Belo Horizonte-MG, 2013.

\begin{tabular}{lcccc}
\hline \multicolumn{1}{c}{ Alimentos } & Dia $\mathbf{1}$ & Dia $\mathbf{2}$ & Dia 3 & Valor p \\
\hline Balcão Quente & 73,0 & 74,0 & 75,0 & 0,872 \\
Arroz & 59,0 & 62,0 & 61,5 & 0,681 \\
Feijão & 73,0 & 76,0 & 76,0 & 0,397 \\
Guarnição & 59,5 & 61,0 & 60,0 & 0,529 \\
Prato Principal 1 & 78,5 & 78,0 & 77,0 & 0,643 \\
Prato Principal 2 & 61,5 & 64,0 & 64,5 & 0,946 \\
Balcão frio & 8,5 & 7,0 & 7,0 & 0,958 \\
Folhosos & 16,0 & 16,0 & 15,5 & 0,099 \\
Legumes & 19,0 & 19,0 & 18,0 & 0,610 \\
\hline
\end{tabular}

As figuras 1 e 2 representam as medianas encontradas para cada preparação quente e fria, respectivamente. Dos restaurantes analisados, seis (60\%) apresentaram inconformidades quanto à temperatura de distribuição dos alimentos. Das preparações quentes, o arroz e o macarrão foram os alimentos que apresentaram as temperaturas inadequadas, e entre as preparações frias, os legumes. As temperaturas dos balcões quente e frio também foram observadas. A temperatura do balcão quente estava abaixo de $80^{\circ} \mathrm{C}$ em sete restaurantes (70\%), e o frio estava abaixo de $10^{\circ} \mathrm{C}$ em todos os restaurantes. 
Tabela 3. Correlação das medianas por horário de distribuição das preparações quentes e firas de restaurantes comerciais da região Centro-Sul de Belo Horizonte-MG, 2013.

\begin{tabular}{lccccc}
\hline \multicolumn{1}{c}{ Alimentos } & H*1 & H2 & H3 & H4 & Valor $\mathbf{~}$ \\
\hline Balcão Quente & 72,0 & 74,5 & 75,0 & 74,5 & 0,598 \\
Arroz & 60,0 & 61,5 & 62,0 & 62,0 & 0,647 \\
Feijão & 75,5 & 76,0 & 76,0 & 75,0 & 0,931 \\
Guarnição & 59,5 & 61,0 & 60,0 & 59,5 & 0,144 \\
Prato Principal 1 & 78,5 & 78,0 & 77,0 & 78,0 & 0,555 \\
Prato Principal 2 & 61,5 & 64,0 & 64,5 & 64,0 & 0,205 \\
Pista Fria & 8,5 & 7,0 & 7,0 & 7,5 & 0,235 \\
Folhosos & 16,0 & 16,0 & 15,5 & 16,0 & 0,343 \\
Legumes & 19,0 & 19,0 & 18,0 & 18,5 & 0,498 \\
\hline
\end{tabular}

$\mathrm{H} 1 *=11: 00, \mathrm{H} 2 *=12: 00, \mathrm{H} 3 *=13: 00, \mathrm{H} 4 *=14: 00$

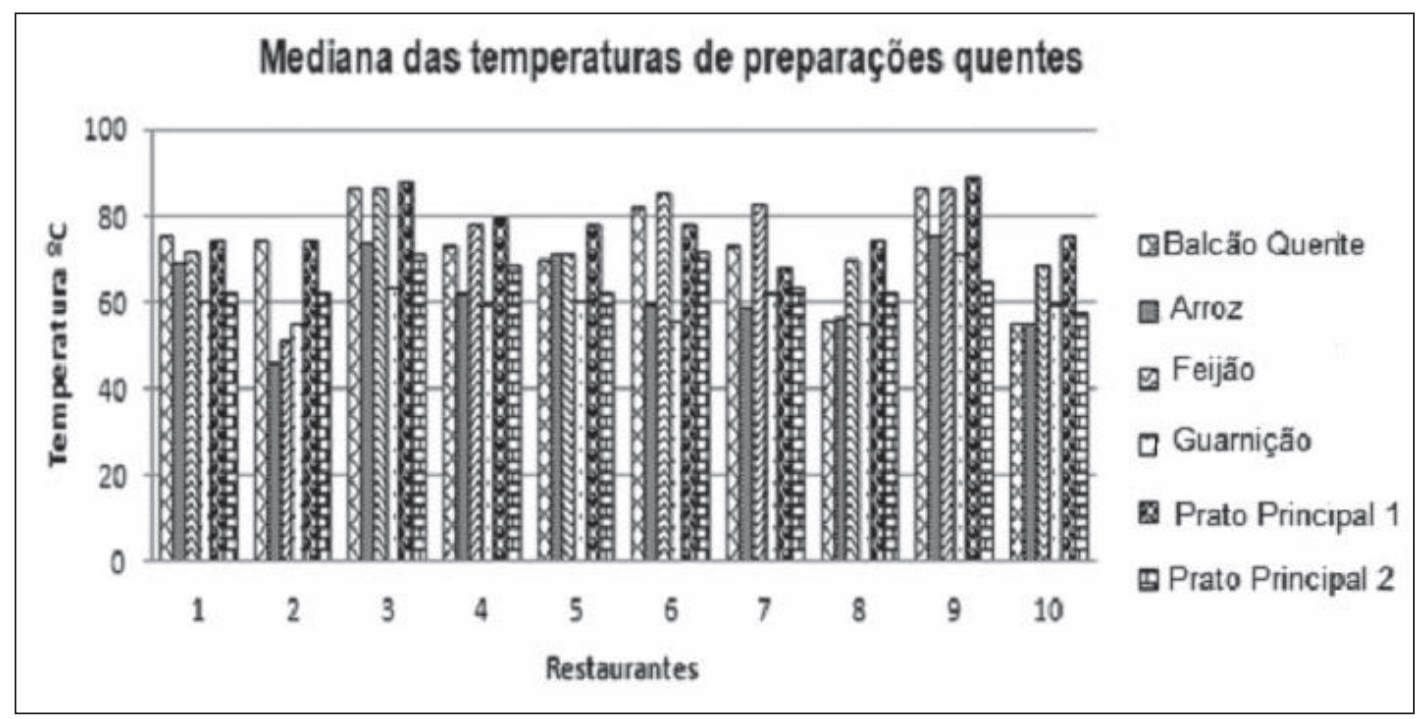

Figura 1. Mediana das temperaturas das preparações quentes em restaurantes comerciais da região Centro-Sul de Belo Horizonte-MG, 2013. 


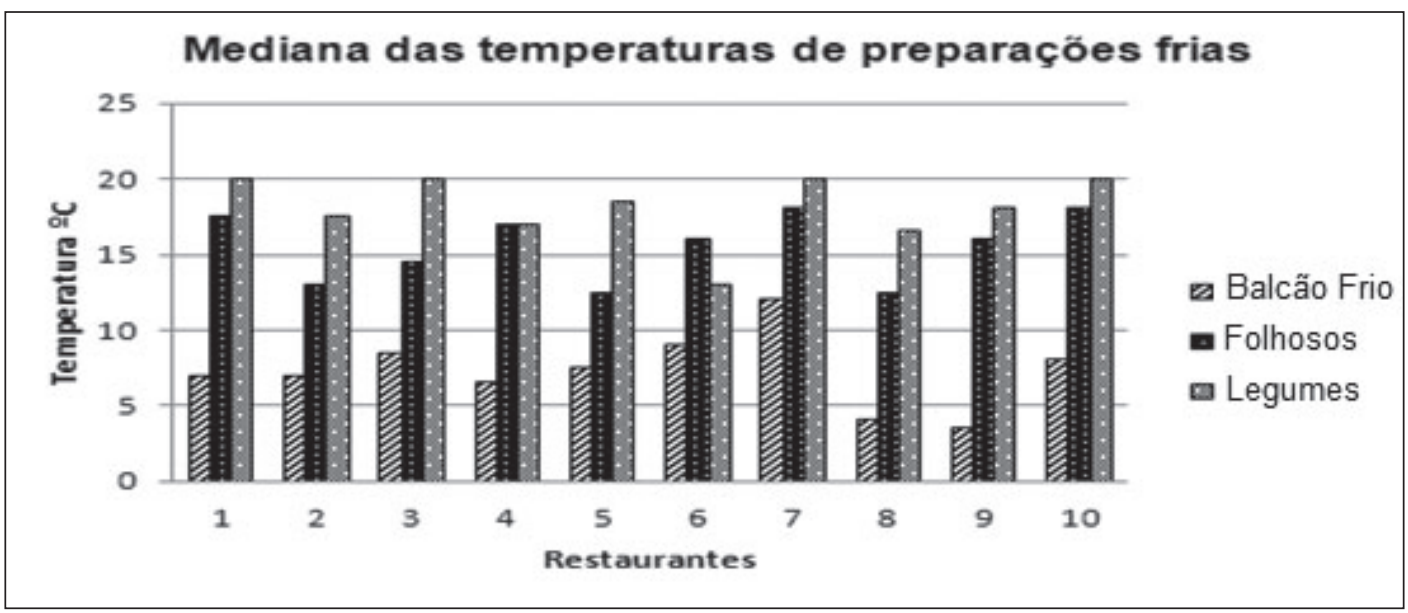

Figura 2. Mediana das temperaturas das preparações frias em restaurantes comerciais da região Centro-Sul de Belo Horizonte-MG, 2013.

\section{Discussão}

$\mathrm{Na}$ análise dos resultados obtidos, verificou-se que apenas quatro restaurantes apresentaram temperaturas adequadas das preparações quentes (figura 1), quando comparadas ao preconizado pela RDC 216/2004. ${ }^{9}$ De acordo com esta resolução, os alimentos, após serem submetidos a cocção, devem ser mantidos em condições de tempo e de temperatura que não favoreçam a multiplicação microbiana, garantindo sua qualidade e segurança. Desse modo, é recomendado que os alimentos sejam submetidos a temperatura superior a 60 por, no máximo, seis horas de exposição. Em relação às preparações frias, observou-se que todas se mantiveram em temperaturas superiores a $10^{\circ} \mathrm{C}$, uma inconformidade de acordo com a referida resolução, que recomenda a manutenção da temperatura dos alimentos frios inferior a $10^{\circ} \mathrm{C}$.

Tanto a Portaria CVS5/2013 ${ }^{9}$ quanto a RDC 216/2004 ${ }^{9}$ preconizam os mesmos parâmetros de temperaturas para as preparações quentes e frias, porém a CVS5/2013 ressalta que se a temperatura das preparações frias ficar entre $10^{\circ} \mathrm{C}$ e $21^{\circ} \mathrm{C}$, pode ser considerada adequada desde que o tempo de exposição não seja superior a duas horas. Diante disto, os alimentos frios observados mostraramse inadequados para o consumo, pois ficaram expostos a temperaturas superiores a $10^{\circ} \mathrm{C}$ por um período de aproximadamente quatro horas, pois não eram armazenados adequadamente após o preparo - na maioria dos restaurantes, as preparações eram mantidas em temperatura ambiente. 
Alves et al. ${ }^{3}$ e Momesso et al. ${ }^{10}$ encontraram resultados semelhantes: $22 \%$ e 20\%, respectivamente, dos alimentos quentes analisados apresentaram temperatura adequada, de acordo com a RDC 216/2004. No entanto, neste trabalho foram encontrados valores mais elevados (40\%) de adequação nas temperaturas.

Ricardo et al., ${ }^{6}$ em estudo realizado em Goiânia, encontraram a temperatura média de $25^{\circ} \mathrm{C}$ para as preparações frias. Segundo a CVS5/2013, deve-se desprezar os alimentos expostos por mais de duas horas acima de $21^{\circ} \mathrm{C}$. No presente estudo, também foram encontradas temperaturas inadequadas, com mediana de 15,5ㄷ $\mathrm{C}$ para folhosos e 18드 para os legumes. A falta de refrigeração adequada após o preparo foi a principal causa destas inconformidades. O tempo de refrigeração pós-preparo não é suficiente para manter o alimento a uma temperatura inferior a $10^{\circ} \mathrm{C}$ - a maioria dos restaurantes não possui pass through ou equipamentos de refrigeração, ou estes não são em número suficiente para armazenar todos os alimentos de forma adequada.

Alves et al. ${ }^{3}$ sugerem que a falha na refrigeração dos alimentos pós-preparo, assim como a falta de conscientização dos manipuladores, são os principais fatores que interferem negativamente no controle de temperatura dos alimentos frios. Além disso, Domene et al. ${ }^{11}$ afirmam que o baixo teor de água nos alimentos dificulta a manutenção da temperatura. Eles observaram que arroz, macarrão e bife foram os alimentos com os piores controles de temperaturas. Ressaltam também que alimentos ricos em amido geralmente não são expostos a temperaturas ideais, já que o aquecimento elevado altera a textura e interfere nas características sensoriais da preparação e, consequentemente, pode diminuir a aceitação.

Apenas três restaurantes apresentaram temperaturas adequadas no balcão térmico, segundo a CVS5/2013, ${ }^{9}$ entre $80^{\circ} \mathrm{C}$ e $90^{\circ} \mathrm{C}$. Os alimentos expostos a temperaturas inadequadas estão mais suscetíveis à multiplicação de microrganismos. ${ }^{8}$

Os principais alimentos, os quais estavam com os piores controles de temperatura, foram arroz, macarrão e bife. As características destas preparações favorecem o crescimento das bactérias Bacillus cereus e coliformes a $45^{\circ} \mathrm{C}$, respectivamente, de acordo com a RDC-12/2001, que em suas atribuições estabelece os padrões microbiológicos sanitários para alimentos. ${ }^{12}$

O Bacillus cereus é uma bactéria mesófila, em forma de bastão, que em ambiente com oxigênio forma esporos. A temperatura mínima de crescimento oscila entre $4^{\circ} \mathrm{C}$ e $5^{\circ} \mathrm{C}$, e máxima, entre $48^{\circ} \mathrm{C}$ e $50^{\circ} \mathrm{C}$. O risco patológico do Bacillus cereus está relacionado à produção de toxinas produzidas pela própria bactéria. Segundo Paiva et al., ${ }^{13}$ as intoxicações ocorrem mediante a conservação inadequada do alimento e devido a falhas no controle do tempo e da temperatura. Essa bactéria pode causar dois tipos de DTA - o diarreico e o emético. No arroz e no macarrão, o mais comum é do tipo emético. ${ }^{14}$ 
Chang et al. ${ }^{15}$ realizaram estudo com preparações a base de arroz frito, em oito restaurantes, a fim de determinar a prevalência de Bacillus cereus nesses pratos. Um de seus objetivos específicos foi analisar a relação tempo e temperatura de exposição no período de produção e consumo. Eles observaram que os alimentos estavam prontos para consumo numa temperatura de $66,1^{\circ} \mathrm{C}$ quando eram preparados depois do pedido, e 59, $8^{\circ} \mathrm{C}$ quando ficavam pré-prontos ou prontos para serem reaquecidos. Houve contaminação por Bacillus cereus em 37,5\% das amostras. Os autores concluíram que o emprego de métodos de processamento, em que é possível controlar o tempo e temperatura, resultou em menor contagem de B. cereus. Os restaurantes analisados no presente trabalho tiveram temperaturas medianas do arroz entre $46^{\circ} \mathrm{C}$ e $75^{\circ} \mathrm{C}$, o que demonstra falha no controle da temperatura.

A temperatura do macarrão de todos os restaurantes analisados estava inadequada, mas permaneceu acima de $50^{\circ} \mathrm{C}$, o que evita o crescimento de B. cereus. E em relação ao arroz, apenas um restaurante apresentou mediana abaixo de $50^{\circ} \mathrm{C}$ (valor de $46^{\circ} \mathrm{C}$ ), indicando risco de crescimento microbiológico e contaminação do alimento.

Passos et al. ${ }^{16}$ realizaram análise de amostra da carne assada devido ao surto de toxinfecção em funcionários de uma empreiteira em São Paulo e encontraram contagem elevada de coliformes, conforme estabelecido pela RDC 12/2001. ${ }^{12}$ De acordo com os autores, a presença de coliformes indica que a preparação está imprópria para o consumo, devido ao risco de toxinfecção alimentar. Isto demonstra que há risco de DTA se não forem monitorados o tempo de exposição e a temperatura das preparações nos restaurantes.

Neste trabalho, embora a temperatura do bife tenha se mostrado inadequada em um restaurante, pode-se afirmar que este alimento não oferecia risco de crescimento de bactérias mesófilas e coliformes, já que a menor mediana esteve em torno de 57ํㅡ. ${ }^{17}$

Fortuna ${ }^{17}$ analisou 22 amostras de carne bovina, antes e após a cocção, e verificou que a cocção adequada suplantou qualquer tipo de contaminação. Já Vieira et al. ${ }^{18}$ analisaram a qualidade microbiológica da carne in natura e de pratos prontos à base de carne servida em Poços de CaldasMG e observaram que a cocção levou a uma redução considerável dos mesófilos aeróbios. Além disso, os autores ressaltam a importância no controle das temperaturas de cocção e distribuição.

Chesca et al. ${ }^{19}$ afirmam que as bactérias presentes na carne são destruídas em parte ou totalmente quando expostas a temperaturas de cocção adequadas, mas o calor não possui efeito residual, isto é, ao final da sua ação, pode ocorrer recontaminação e/ou multiplicação. Por este motivo, os alimentos submetidos ao calor devem ser consumidos de imediato ou conservados em temperaturas adequadas. 


\section{Conclusão}

Os resultados da análise das medianas das temperaturas dos diferentes restaurantes mostraram que as maiorias dos restaurantes não controlam nem monitoram de modo adequado a relação existente entre o tempo de exposição e a temperatura das preparações. A falta de mão de obra treinada, equipamentos e espaço físico são os principais motivos pelas falhas no controle do binômio tempo-temperatura. Consequentemente, há grande risco de contaminação microbiológica patogênica, com possível risco de surto de toxinfecção de origem alimentar.

Para que a possibilidade da ocorrência de DTA seja diminuída nos restaurantes, sugere-se a implantação de técnicas de controle de temperatura mais efetivas, com capacitação adequada dos profissionais envolvidos nesta ação.

\section{Agradecimentos}

Aos proprietários e funcionários dos restaurantes que participaram deste estudo.

\section{Referências}

1. Akutsu RC, Botelho RA, Camargo EB, Sávio KEO, Araújo WC. Adequação das boas práticas de fabricação em serviços de alimentação. Rev. Nutr. 2005; 18(3):419-27.

2. Zandonadi RP, Botelho RBA, Sávio KEO, Akutsu RC, Araújo WMC. Atitudes de risco do consumidor em restaurantes de auto-serviço. Rev. Nutr. 2007; 20(1):19-26.

3. Alves MG, Ueno M. Restaurantes self-service: segurança e qualidade sanitária dos alimentos servidos. Rev. Nutr. 2010; 23(4):573-80.

4. Associação Brasileira de Refeições Coletivas. Valor econômico faz um raio x do mercado de refeições coletivas. Boletim Notícias ABERC 2012;(124):3. [acesso em: 04 dez. 2012]. Disponível em: http:// www.aberc.com.br/Uploads/pdfs/Jornal_ABERC-124.pdf

5. Fundação Instituto Brasileiro de Geografia e Estatística. Pesquisa de orçamentos familiares 20082009: despesas, rendimentos e condições de vida. Rio de Janeiro: IBGE; 2010; 62-67.

6. Ricardo FO, Morais MP, Carvalho ACMS. Controle de tempo e temperatura na produção derefeições de restaurantes comerciais na cidade de Goiânia-GO. Demetra: Nutr e Saúde. 2012; 7(2):85-96.

7. Marinho CB, Souza CS, Ramos SA. Avaliação do binômio tempo-temperatura de refeições transportadas. Rev e-scientia. 2009; 2(1):1-11.

8. Brasil. Resolução RDC n. 216, de 15 de set. 2004. Regulamento Técnico de Boas Práticas para Serviços de Alimentação. Diário Oficial da União, 16 set. 2004. 
9. São Paulo. Portaria CVS 5/2013, de 09 de abril de 2013. Regulamento Técnico, que estabelece os Parâmetros e Critérios para o Controle Higiênico-Sanitário em Estabelecimentos de Alimentos. Diário Oficial do Estado, 19 abr. 2013; Seção 1:32-35.

10. Momesso AP, Matté MH, Germano PML. Avaliação das condições higiênico-sanitárias, por quilo, do município de São Paulo, durante o período de distribuição de refeições. Hig. Aliment. 2005; 19(136):81-9.

11. Domene SMA. Técnica dietética: teoria e aplicações. Rio de Janeiro: Guanabara Koogan; 2011. 249p.

12. Brasil. Resolução RDC n. 12, 02 de jan. 2001. Regulamento Técnico sobre Padrões Microbiológicos para Alimentos. Diário Oficial da União 10 jan. 2001.48p.

13. Paiva EP, Fai AEC, Soares DS, Stamford TLM. Bacilluscereus e suas toxinas em alimentos. Hig. Aliment. 2009; 23(170/171):87-92.

14. Abee T, Groot MN, Tempelaars M, Zwietering M, Moezelaar R, Voort MVD. Germination and outgrowth of spores of Bacillus cereus group members: diversity and role of germinant receptors. Food Microbiol. 2010; 28(2):199-208.

15. Chang H, Lee J, Han B, Kwaky T, Kim J. Prevalence of the levels of Bacillus cereus in fried rice dishes and its exposure assessment from Chinese-style Restaurants. Food Sci. Biotechnol. 2011; 20(5):1351-59.

16. Passos EC, Almeida CS, Rosa JP, Rozman LM, Mello ARP, Souza CV, Paschoal RC, Tavares M. Surto de toxinfecção alimentar em funcionários de uma empreiteira da construção civil no município de Cubatão, São Paulo/Brasil. Rev. Inst. Adolfo Lutz 2008; 67(3):237-40.

17. Fortuna JL. Aspectos higiênico-sanitário no preparo de carne bovina servida em refeições escolares de instituições escolares municipais e estaduais, no Estado do Rio de Janeiro. Hig. Aliment. 2002; 16(95):23-33.

18. Vieira CRN, Silva RR, Martino HSD, Chavasco JK. Qualidade microbiológica da merenda escolar servida nas escolas estaduais de Poços de Caldas, MG. Hig. Aliment. 2005; 19(128):90-4.

19. Chesca AC, Caetano AM, Leite APC, Polveiro AM, Terra AD, Lyra FS, et al. Avaliação das temperaturas de pistas frias e pistas quentes em restaurantes da cidade de Uberaba, MG. Hig. Aliment. 2001; 15(87):38-43.

Recebido: $26 / 2 / 2015$

Revisado: $14 / 4 / 2015$

Aprovado: 25/4/2015 